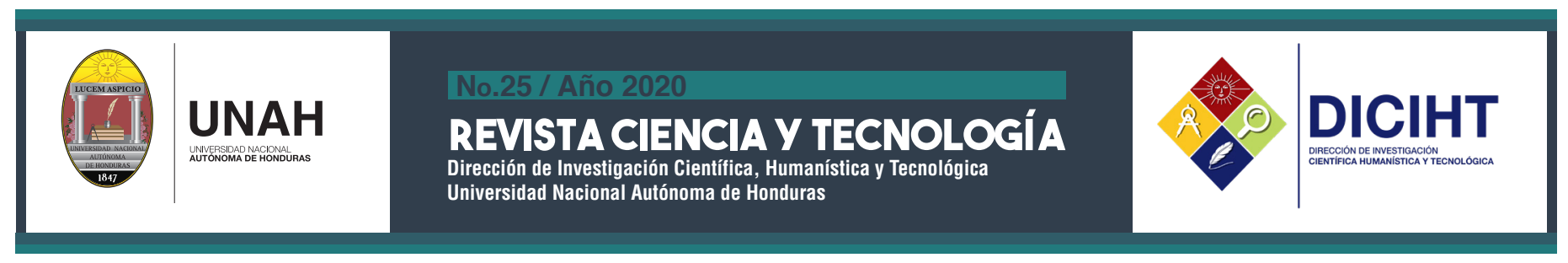

ARTICULOORICINAL

\title{
Evaluación de la tasa de consumo de oxígeno del Penaeus vannamei con relación a la salinidad, temperatura y peso corporal
}

\section{Assessment of the oxygen consumption rate of the Penaeus vannamei with respect to salinity, temperature and body weight}

\section{Édgar Osiris Carranza}

a Departamento de Acuacultura y Biología Marina, Centro Universitario Regional del Litoral Pacífico, Universidad Nacional Autónoma de Honduras, Choluteca, Honduras

DOI 10.5377/rct.v13i25.10412 Recibido: 17 febrero 2020 Aceptado: 03 de agosto 2020 Publicado en línea: 23 noviembre 2020

\section{RESUMEN}

El oxígeno disuelto (OD) es el parámetro de mayor importancia en acuicultura. En el cultivo del camarón, una baja solubilidad de oxígeno afecta el crecimiento y la salud de los organismos. La disponibilidad del OD depende de la respiración del fondo del estanque, el fitoplancton y el cultivo. El objetivo del estudio fue determinar el consumo de oxígeno del camarón en las salinidades de 4, 12, 22 y 32 UPS, las temperaturas de 28 y $31^{\circ} \mathrm{C}$, y en los pesos de 4, 6, 8, 10, 12 y $14 \mathrm{~g}$. Se registró la concentración inicial de OD a los 10, 20, 30 y 40 minutos para obtener el consumo relativo $(\mathrm{Cr})$ de OD, consumo absoluto (CA) según el tiempo de exposición y el consumo porcentual (CP). Se manejó un diseño factorial de 6 x 4 x 6 × 2 y la prueba Tukey para la comparación de medias. El mayor consumo de oxígeno se observó en las salinidades de 32 UPS $(\mathrm{P}<0.0001)$ y a $31^{\circ} \mathrm{C}(\mathrm{P}<0.0002)$. Los organismos de 14 y $12 \mathrm{~g}$ de peso fueron los que consumieron más OD $(\mathrm{P}<0.0001)$; a los primeros 10 minutos, los camarones llegan a un CP mayor del $40 \%$ de OD disponible.
La temperatura y la salinidad influyen en la tasa de respiración; también, los organismos de mayor tamaño tienen más demandan energética y $\mathrm{Cr}$ de OD.

Palabras clave: metabolismo, oxígeno disuelto, salinidad, tasa de respiración

\section{ABSTRACT}

Dissolved oxygen (DO) is the most important parameter in aquaculture. In shrimp farming, low oxygen solubility affects growth and health of organisms. The availability of DO depends on pond bottom respiration, phytoplankton and culture. The objective of the study was to determine the oxygen consumption of shrimp in the salinity of $4,12,22$ and 32 UPS, temperatures of 28 and $31^{\circ} \mathrm{C}$, and in the weights of 4, 6, 8, 10, 12 and $14 \mathrm{~g}$. The concentration of OD was recorded at 10, 20, 30 and 40 minutes to obtain the relative consumption $(\mathrm{Cr})$ of $\mathrm{OD}$, absolute consumption (AC) according to exposure time and percentage consumption (PC). A $6 \times 4 \times 6$

\footnotetext{
Autor corresponsal

$\triangle$ Édgar Osiris Carranza

edgar.carranza@unah.edu.hn
} 
x 2 factorial design and the Tukey test of means were used. The highest oxygen consumption was observed at salinities of 32 UPS $(\mathrm{P}<0.0001)$ and at $31{ }^{\circ} \mathrm{C}$ $(\mathrm{P}<0.0002)$. Organisms weighing 14 and $12 \mathrm{~g}$ were those that consumed the most $\mathrm{OD}(\mathrm{P}<0.0001)$; within the first 10 minutes, the shrimps reached a $\mathrm{CP}$ greater than $40 \%$ of available OD. Temperature and salinity influence the breathing rate; also, larger organisms have more energy and $\mathrm{Cr}$ demands of OD.

Keywords: metabolism, dissolved oxygen, salinity, respiration rate

\section{INTRODUCCIÓN}

En Honduras, la acuicultura es una actividad económica importante, siendo la región del Golfo de Fonseca en donde se desarrolla el rubro del camarón. El área de cultivo comprende 22,748 hectáreas de espejo de agua, manteniendo en producción 20,240 hectáreas y distribuidas en 404 proyectos camaroneros entre productores artesanales, pequeños, medianos y grandes corporaciones (FIDE 2017). Las exportaciones se mantienen anualmente en constante crecimiento, debido al mejoramiento de las técnicas en el sistema de cultivo, lo que permite generar alrededor de 216 millones de dólares anuales en divisas para el país (Rodríguez 2019). Esta actividad ha tenido un impacto económico y social para el desarrollo en la región. Desde los inicios de la camaronicultura, el sistema de producción semi-intensivo es el que más se desarrolla en las granjas de cultivo, siendo un desafío para los productores el manejo de la calidad de agua y la concentración de oxígeno.

El oxígeno disuelto (OD) es uno de los factores más importantes en la calidad de agua en acuicultura. En el cultivo del camarón, una baja concentración de oxígeno afecta el crecimiento y la salud de los organismos (Supriatna et al. 2017). La concentración del oxígeno depende de propiedades físicas, químicas y bioquímicas del cuerpo de agua. Cuando se presenta una baja concentración de OD, se altera la calidad de agua, llegando a provocar una baja conversión alimenticia y altas mortalidades (Galand et al. 2019). Para un acuicultor, es importante manejar adecuadamente la calidad de agua y la forma de ingresar oxígeno al estanque.

Las fuentes que proveen OD son la fotosíntesis, la difusión del oxígeno atmosférico en la superficie del agua, el recambio de agua y el uso de aireadores mecánicos. De estos, el recambio y la incorporación de aireadores son utilizados en forma preventiva o correctiva en la producción acuícola para cumplir con la demanda de OD de la columna de agua (Boyd 1998). El oxígeno se incrementa durante el día por el proceso natural de la fotosíntesis, llegando hasta la concentración de $14 \mathrm{mg} / \mathrm{L}$ en las horas de mayor radiación solar, pero durante la noche, el OD baja por el proceso de la respiración del estanque.

El OD regula procesos bioquímicos de la calidad del agua como la nitrificación catiónica, la desnitrificación y la degradación de la materia orgánica. La disponibilidad de OD en el estanque también depende de la tasa de respiración de los organismos acuáticos, las bacterias aeróbicas y la materia orgánica durante el proceso de descomposición e incorporación de nutrientes en el fondo (Carranza 2018). Entre el 50 y $55 \%$ del OD disponible es consumido por la respiración del fondo, seguido por el fitoplancton que utiliza entre el 40 al $45 \%$, y la respiración del cultivo que consume el $5 \%$ del OD (Boyd y Tucker 1992). La tasa de consumo del OD en los camarones depende de factores ambientales y su peso.

Los factores ambientales, como la temperatura y la salinidad, influyen para que se presente mayor variabilidad en el consumo de oxígeno por los camarones (Valenzuela-Quiñónez et al. 2011) e influye en los procesos biológicos, mientras que la 
salinidad incide en la demanda osmorregulatoria del camarón (Yan et al. 2007). La concentración del oxígeno se reduce al aumentar la salinidad y la temperatura del agua, y estos factores combinados pueden reducir la concentración del OD hasta en un $65 \%$ (Boyd 2014). En acuícultura, se debe conocer la capacidad de respiración del estanque y sobre todo de los camarones, para proveer la cantidad adecuada de oxígeno al presentarse episodios de hipoxia o anoxia.

El peso del camarón es un factor que influye en el consumo de OD. En animales de menor tamaño, el consumo es mayor porque demandan una mayor cantidad de energía para el desarrollo de sus procesos metabólicos (Suárez Álvarez y Oquendo Pérez 2012). El objetivo del estudio consistió en determinar el consumo de oxígeno del camarón en las salinidades de $4,12,22$ y 32 UPS, temperaturas entre 27 y $31^{\circ} \mathrm{C}$, y en los pesos de $4,6,8,10,12$ y $14 \mathrm{~g}$, para estimar los requerimientos de oxígeno bajo diferentes condiciones de salinidad, temperatura y peso.

\section{MATERIALES Y MÉTODOS}

Este estudio se realizó en el laboratorio húmedo del Centro Universitario Regional del Litoral Pacífico (CURLP) ubicado en la cuidad de Choluteca, en el sur de Honduras, bajo condiciones de manejo controladas. Se utilizaron 288 camarones con pesos de 4, 6, 8, 10, 12 , y $14 \mathrm{~g}$, distribuidos en 48 animales por peso. Estos fueron obtenidos de una finca de cultivo ubicada a 46 $\mathrm{km}$ al sur oeste del CURLP y se transportaron en un tanque de $1 \mathrm{~m}^{3}$ con aireación constante. Al llegar al laboratorio húmedo, los camarones se mantuvieron en un período de adaptación por 72 horas antes de iniciar el proceso de aclimatación, en un tanque de $5 \mathrm{~m}^{3}$. Para mantener la calidad de agua, cada 96 horas se realizó un recambio total y se utilizó agua marina filtrada tratada en un laboratorio de producción de postlarva. Diariamente, se alimentó con una dieta balanceada al
$35 \%$ de proteína aplicada a la razón del $3 \%$ del peso vivo y distribuida en tres raciones durante el día.

\subsection{Diseño del experimento}

Para observar el consumo de oxígeno, se manejaron 36 recipientes cilíndricos de polietileno trasparentes con cierre hermético y de boca ancha con 1 litro de capacidad. Estos se pintaron externamente de color negro con el propósito de impedir el ingreso de la luz, evitando la fotosíntesis. Se colocaron dos recipientes por tubo en 18 tubos de policloruro de vinilo (PVC) de $1.2 \mathrm{~m}$ de longitud y $0.25 \mathrm{~m}$ de diámetro, sujetados con alambre de amarre a la mitad de la altura del tubo PVC a $0.6 \mathrm{~m}$ del piso.

Cada recipiente se llenó con un litro de agua marina filtrada a la salinidad que estaban los camarones, y se colocó un camarón por recipiente. Una vez llenos, los recipientes se cerraron herméticamente para sumergirlos junto con el tubo de PVC en un tanque circular de geomembrana con $1.3 \mathrm{~m}$ de altura y $5 \mathrm{~m}^{3}$ de volumen, registrando cada 10 minutos el consumo de oxígeno disuelto hasta llegar a los 40 minutos. Los camarones, después de finalizada la prueba, fueron sacrificados.

De los 36 recipientes utilizados, se depositaron en ellos camarones con los pesos de 4, 6, 8, 10, 12 y $14 \mathrm{~g}$, manejando seis repeticiones por cada peso. Para evaluar el efecto de la temperatura, también se registró el consumo de oxígeno a las 2:00 pm, por estar dentro de las horas de mayor radicación solar y temperatura; a esa hora, la temperatura promedio fue de $31^{\circ} \mathrm{C}$ y por la noche, a las $9 \mathrm{pm}$, fue de $27^{\circ} \mathrm{C}$. Para observar el efecto de la salinidad, se manejaron las concentraciones de 4, 12, 22 y 32 unidades prácticas de sal (UPS). En cada concentración de salinidad, se usaron 72 camarones distribuidos en los seis tamaños, tres repeticiones y dos temperaturas $\left(31\right.$ y $\left.27^{\circ} \mathrm{C}\right)$. 


\subsection{Intervenciones}

Inicialmente, los camarones se encontraron a 4 UPS y gradualmente se incrementó 1 UPS por día. Para aumentar la salinidad de 4 a 12 UPS, este proceso de aclimatación se realizó durante 8 días; al noveno día, se recolectaron los animales necesarios para hacer las mediciones de OD en los recipientes. Con los camarones restantes, se incrementó progresivamente la salinidad de 12 a 22 UPS durante 10 días continuos; al onceavo día, se registraron nuevas mediciones con otro grupo de 72 camarones de OD, y para observar el consumo de OD a 32 UPS se manejó el mismo procedimiento anterior con los camarones restantes. Durante todo este proceso, no se registraron mortalidades.

El consumo de oxígeno de los camarones se midió por medio de un medidor de oxígeno de la marca YSI550A que despliega simultáneamente temperatura en el rango de -5 a $45{ }^{\circ} \mathrm{C}$, OD entre 0 y $20 \mathrm{mg} / \mathrm{L}$ y el porcentaje de saturación de aire de 0 a $200 \%$, contando con un sensor polarográfico no desmontable y calibrado a las condiciones ambientales de la zona (YSI 2002). Se registró la concentración inicial de OD de los recipientes y a los 10, 20, 30 y 40 minutos de exposición dentro del tanque; con esta información, se obtuvo el consumo relativo $(\mathrm{Cr})$ de $\mathrm{OD}$, consumo absoluto (CA) según el tiempo de exposición y el consumo porcentual (CP). Para el consumo de oxígeno, se utilizaron las siguientes ecuaciones:

$$
\begin{gathered}
C r=O D_{\text {inicial }}-O D_{\text {final }} \\
C A=C r+N \\
C P=\frac{C r}{C A_{\text {total }}}
\end{gathered}
$$

Donde $\mathrm{N}$ es el Cr anterior del tiempo de exposición.

\subsection{Análisis estadísticos}

Se utilizó un diseño factorial de $6 \times 4 \times 6 \times 2$ para encontrar diferencias estadísticas en el consumo relativo de oxígeno de los camarones sometidos a las combinaciones de las cuatro salinidades, los seis pesos corporales, y la temperatura. De igual manera, se manejó este mismo diseño para el consumo absoluto y consumo porcentual de oxígeno. Los valores porcentuales transformados se obtuvieron mediante la expresión:

$$
y=\operatorname{arcsen} \sqrt{ } x / 100
$$

En la comparación múltiple de medias, se usó la prueba Tukey al 0.05 de nivel de significancia. Todos estos análisis fueron realizados con el programa estadístico Infostat ${ }^{\circledR} 2018$.

\section{RESULTADOS}

\subsection{Consumo relativo de OD}

Se observaron diferencias estadísticas $(\mathrm{P}<0.0001)$ en el consumo de oxígeno de los camarones a los 10 , 20, 30 y 40 minutos de exposición sin una fuente de aireación exógena. Se encontraron también diferencias significativas en el oxígeno consumido en las cuatro combinaciones de salinidad $(\mathrm{P}<0.0001)$ y en el consumo del oxígeno influenciado por la temperatura $(\mathrm{P}<0.0002)$. En las salinidades, se observó el mayor consumo a los 10 minutos en los camarones que estuvieron a 32 UPS con $2.31 \mathrm{mg} / \mathrm{L}$ de OD. Este valor fue estadísticamente diferente al consumo que mostraron los camarones a 4 UPS con $1.43 \mathrm{mg} / \mathrm{L}$. A los 40 minutos de respiración, el consumo de oxígeno se redujo a $0.33 \mathrm{mg} / \mathrm{L}$ en los organismos a 32 UPS, y a $0.22 \mathrm{mg} / \mathrm{L}$ en los que estuvieron a 4 UPS. En general, el oxígeno consumido en promedio después de los 40 minutos fue de $3.16 \mathrm{mg} / \mathrm{L}$ para la salinidad de 4 UPS, $3.28 \mathrm{mg} / \mathrm{L}$ en $12 \mathrm{UPS}, 3.53 \mathrm{mg} / \mathrm{L}$ en 22 UPS y 3.71 
$\mathrm{mg} / \mathrm{L}$ para los organismos que estuvieron a 32 UPS.

Durante el día, la temperatura del agua fue $4{ }^{\circ} \mathrm{C}$ mayor que por la noche y se reflejó en el consumo del OD en los camarones. A los 10 minutos, el OD consumido a $31{ }^{\circ} \mathrm{C}$ fue de $1.84 \mathrm{mg} / \mathrm{L}$ y a $27{ }^{\circ} \mathrm{C}$ este valor fue de $1.36 \mathrm{mg} / \mathrm{L}$. A los 40 minutos, se observó que a $31^{\circ} \mathrm{C}$, el OD consumido fue de $0.28 \mathrm{mg} / \mathrm{L}$ y a $27{ }^{\circ} \mathrm{C}$, el oxígeno consumido fue de $0.43 \mathrm{mg} / \mathrm{L}$. El mayor consumo ocurrió en los primeros minutos; al llegar a los 40 minutos, la cantidad disponible de OD en el medio fue la que se observó en el consumo de los camarones.

En los pesos de los camarones, se encontraron diferencias $(\mathrm{P}<0.0001)$. Los que más consumieron oxígeno en los primeros 10 minutos fueron los de 14 gramos de peso, seguido por los de 4 y 6 gramos (Fig. 1). Pero este consumo se reduce al incrementar el tiempo a 20, 30 y 40 minutos.
Los organismos de $4 \mathrm{~g}$, a los 10 minutos respiraron $1.85 \mathrm{mg} / \mathrm{L}$ de OD y finalizaron con $0.22 \mathrm{mg} / \mathrm{L}$ a los 40 minutos, siendo este valor acumulado de $3.19 \mathrm{mg} / \mathrm{L}$. En los camarones de 8 y $10 \mathrm{~g}$, se observó un consumo inicial de 1.66 y $1.73 \mathrm{mg} / \mathrm{L}$ a los 10 minutos, llegando a los 40 minutos a 0.37 y $0.34 \mathrm{mg} / \mathrm{L}$ de oxígeno, respectivamente. En los organismos de 12 y $14 \mathrm{~g}$, a los 10 minutos la respiración del oxígeno fue de 1.76 y $2.05 \mathrm{mg} / \mathrm{L}$ y a los 40 minutos, este consumo llegó a 0.44 y $0.33 \mathrm{mg} / \mathrm{L}$; la respiración del oxígeno en total llegó a 3.91 y $3.76 \mathrm{mg} / \mathrm{L}$ después de los 40 minutos.

\subsection{Consumo absoluto de OD}

El consumo absoluto corresponde al consumo del oxígeno después de $10,20,30$ y 40 minutos de respiración. En las combinaciones de salinidad y temperaturas, se observaron diferencias estadísticas $(\mathrm{P}<0.0001)$. Al igual que en el consumo relativo de

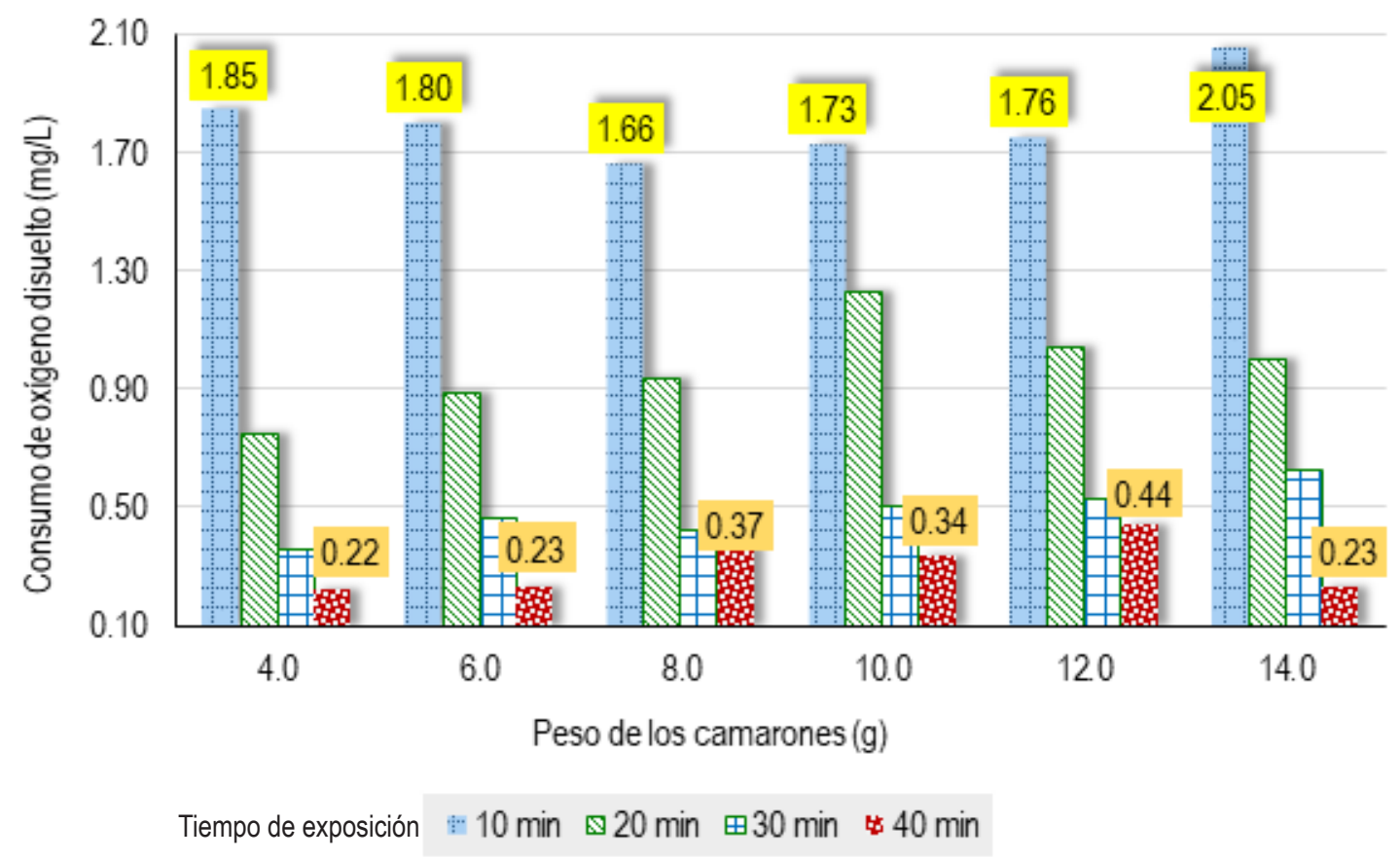

Fig. 1 Consumo individual de OD en camarones con pesos de 4, 6, 8, 10, 12 y 14 g manejados a 10, 20, 30 y 40 min expuestos a un medio en ausencia de aireación externa 
OD, la temperatura y la concentración de salinidad influye en el consumo de OD. Los camarones que estuvieron en el medio a 32 UPS a los 10 minutos, el OD respirado fue de $2.31 \mathrm{mg} / \mathrm{L}$ y se incrementó a 3.71 $\mathrm{mg} / \mathrm{L}$ a los 40 minutos; en cambio, los organismos a 4 UPS a los 10 minutos, el consumo de OD fue de 1.43 $\mathrm{mg} / \mathrm{L}$ y finalizó en $3.16 \mathrm{mg} / \mathrm{L}$.

Al comparar el consumo absoluto de OD según las combinaciones de los pesos de los camarones, también se encontraron diferencias altamente significativas $(\mathrm{P}<0.0001)$. Los camarones que mostraron el mayor consumo de OD fueron los que tuvieron pesos de 14 gramos, seguido por los camarones de 10 y 12 gramos (Fig. 2). A los 10 minutos, se observó un consumo inicial entre 1.76 y $2.5 \mathrm{mg} / \mathrm{L}$ en las seis combinaciones de pesos. A los 20 minutos, el consumo, por ser acumulado, se incrementó entre 2.6 y $3.05 \mathrm{mg} / \mathrm{L}$. A los 30 minutos, el consumo fue entre 2.96 y $3.67 \mathrm{mg} / \mathrm{L}$, y a los 40 minutos, el consumo final en promedio fue entre 3.18 y $3.91 \mathrm{mg} / \mathrm{L}$.

\subsection{Consumo porcentual de OD}

En el consumo porcentual, se encontraron diferencias significativas en las combinaciones de salinidad $(\mathrm{P}<0.0001)$, temperatura $(\mathrm{P}<0.00001)$ y los pesos de los camarones $(\mathrm{P}<0.0002)$, presentando similar comportamiento al consumo absoluto de OD. En los camarones de $14 \mathrm{~g}$, se observó el consumo del $98 \%$ del OD después de los 40 minutos de respiración. En los de $12 \mathrm{~g}$, este valor fue del $94 \%$, al igual que los organismos de $10 \mathrm{~g}$. En los camarones con pesos de 6 y 8 g, se observó que el consumo de OD fue del 85 $\%$, y con los de 4 g llegó al $80 \%$. Porcentualmente, la mayor tasa de respiración ocurre en los primeros 10 minutos consumiendo entre el 46 y $51 \%$ de OD disponible en el cuerpo de agua (Fig. 3), y este consumo se incrementa al aumentar el tiempo de respiración hasta llegar a valores críticos que producen la muerte en los organismos.

A los 20 minutos de respiración, el OD consumido fue entre el 19 y $25 \%$, a los 30 minutos el consumo de oxígeno observado fue entre el 9 y $14 \%$, y a los

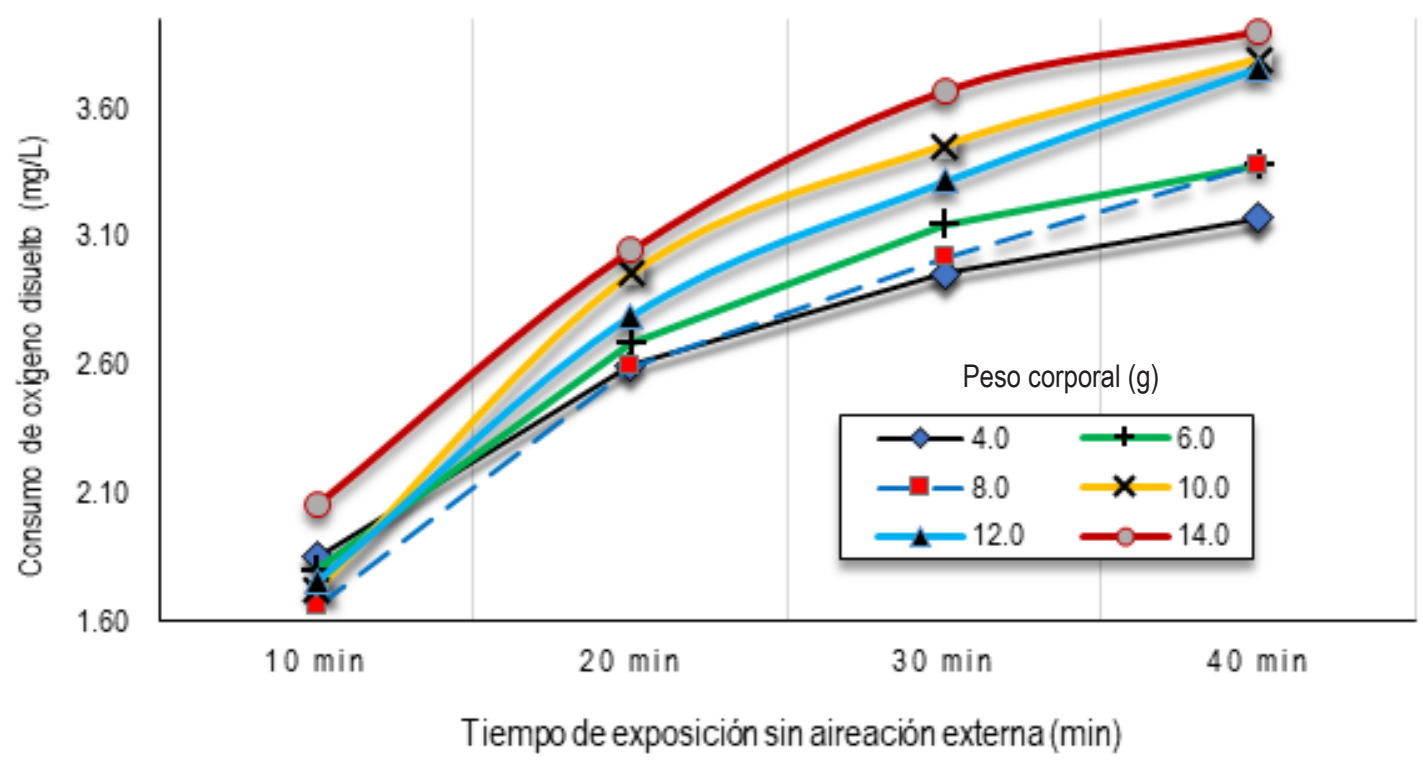

Fig. 2 Consumo acumulado de OD en camarones con pesos de 4, 6, 8, 10, 12 y 14 g manejados a 10, 20, 30 y 40 min expuestos a un medio en ausencia de aireación externa

60 


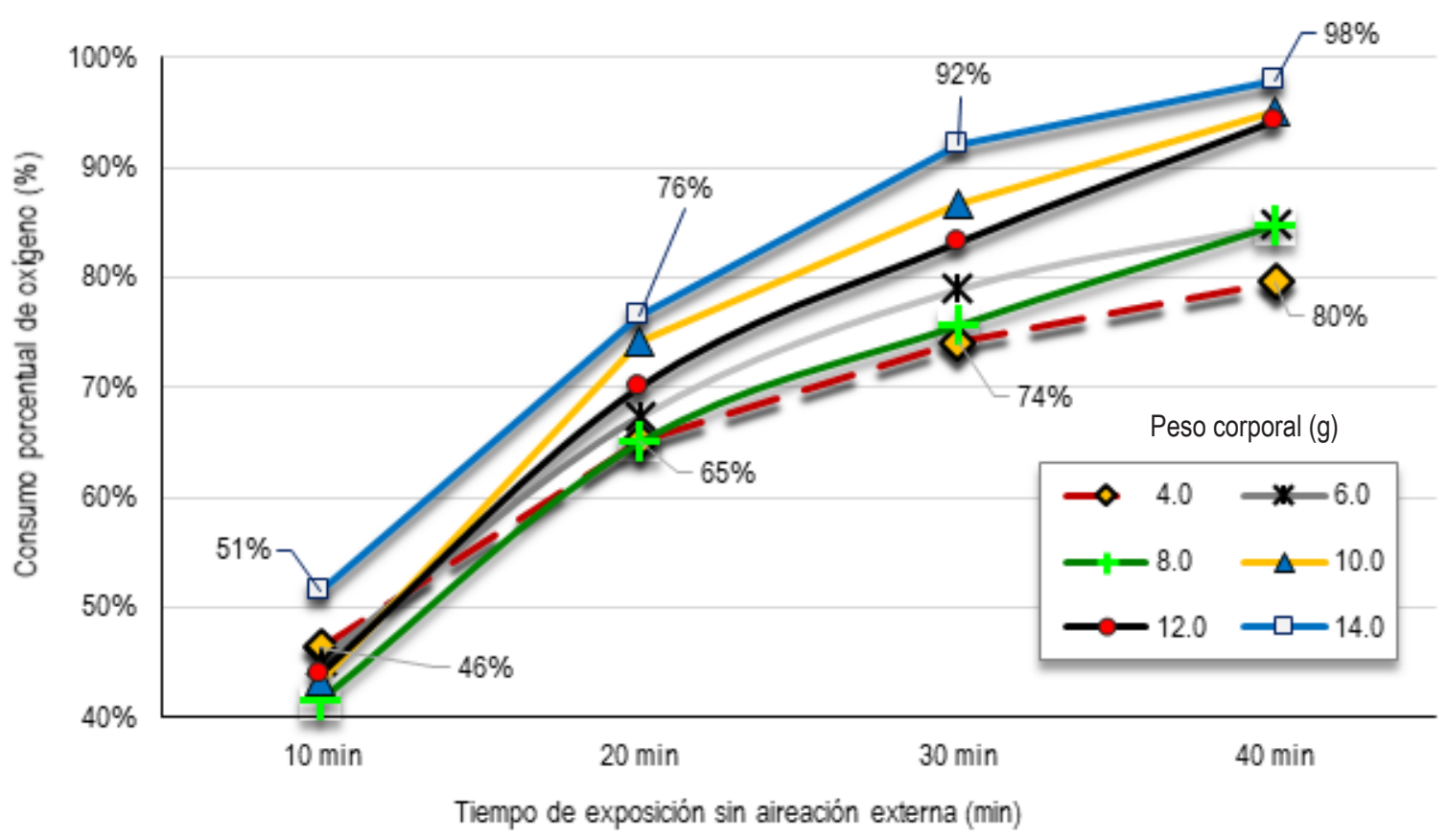

Fig. 3 Consumo en porcentaje de OD en camarones con pesos de 4, 6, 8, 10, 12 y 14 g manejados a 10, 20, 30 y 40 min expuestos a un medio en ausencia de aireación externa

40 minutos la respiración fue entre el 6 y $7 \%$. Al incrementar los minutos de respiración, la cantidad de OD se reduce porque baja la disponibilidad del oxígeno en el medio y los organismos disminuyen su metabolismo. En general, los organismos de mayor salinidad, mayor temperatura y peso fueron los que en forma acumulada consumieron casi todo el OD disponible.

\section{DISCUSIÓN}

Se observó un mayor consumo de OD en los camarones que fueron manejados a $31{ }^{\circ} \mathrm{C}$. Esto se debe a que la temperatura acelera el metabolismo y la tasa de respiración (Suárez Álvarez y Oquendo Pérez 2012). Influye también la disponibilidad del OD, ya que al incrementar la temperatura, la concentración del OD disminuye (Boyd 1990). La máxima concentración de OD que se puede encontrar en el agua es de 14.7 $\mathrm{mg} / \mathrm{L}$, pero depende del contacto del agua con el aire, la saturación de vapor de agua en el aire, factores ambientales como la presión barométrica a diferentes altitudes, la salinidad y la temperatura (Boyd 2014).

La concentración de la salinidad afecta la tasa de respiración. En salinidades más bajas, existe un menor consumo de oxígeno debido a que el proceso metabólico se reduce al igual que la osmorregulación, demandando un menor consumo energético (Tabla 1). En general, la tasa de respiración está relacionada directamente proporcional a la temperatura y la salinidad. Ponce et al. (2013) encontraron que estos organismos tienen la capacidad de adaptar su tasa de respiración a los cambios de temperatura y salinidad.

El peso de los camarones también es un factor que influye en el consumo de OD. En organismos con un menor peso, el consumo de OD es menor en comparación con los organismos de mayor tamaño (Tabla 1), a pesar de que la tasa metabólica es más 
Tabla 1 Consumo promedio de oxígeno disuelto en $\mathrm{mg} / \mathrm{L}$ de camarones manejados a diferentes pesos y salinidades durante cuatro tiempos de exposición sin aireación externa

\begin{tabular}{|c|c|c|c|c|c|c|}
\hline \multirow{2}{*}{$\begin{array}{l}\text { Peso } \\
(\mathrm{g})\end{array}$} & \multirow{2}{*}{$\begin{array}{l}\text { Salinidad } \\
\qquad(\mathrm{g} / \mathrm{L})\end{array}$} & \multicolumn{4}{|c|}{ Tiempo (min) } & \multirow{2}{*}{$\bar{x}$} \\
\hline & & 10 & 20 & 30 & 40 & \\
\hline 4 & \multirow{6}{*}{4} & 1.36 & 0.62 & 0.43 & 0.14 & \multirow{6}{*}{0.79} \\
\hline 6 & & 1.35 & 0.91 & 0.44 & 0.16 & \\
\hline 8 & & 1.29 & 1.40 & 0.47 & 0.20 & \\
\hline 10 & & 1.45 & 1.24 & 0.45 & 0.24 & \\
\hline 12 & & 1.56 & 0.90 & 0.53 & 0.27 & \\
\hline \multirow[t]{2}{*}{14} & & 1.54 & 1.12 & 0.57 & 0.33 & \\
\hline & $\bar{X} \pm S$ & $1.43 \pm 0.11$ & $1.03 \pm 0.28$ & $0.48 \pm 0.06$ & $0.22 \pm 0.07$ & \\
\hline 4 & \multirow{6}{*}{12} & 1.31 & 0.59 & 0.25 & 0.33 & \multirow{6}{*}{0.82} \\
\hline 6 & & 1.35 & 1.13 & 0.55 & 0.19 & \\
\hline 8 & & 1.65 & 0.84 & 0.24 & 0.54 & \\
\hline 10 & & 1.91 & 0.83 & 0.44 & 0.46 & \\
\hline 12 & & 1.14 & 1.11 & 0.66 & 0.50 & \\
\hline \multirow[t]{2}{*}{14} & & 2.31 & 0.75 & 0.45 & 0.14 & \\
\hline & $\bar{X} \pm S$ & $1.61 \pm 0.44$ & $0.88 \pm 0.21$ & $0.43 \pm 0.17$ & $0.36 \pm 0.17$ & \\
\hline 4 & \multirow{6}{*}{22} & 2.37 & 0.89 & 0.38 & 0.20 & \multirow{6}{*}{0.88} \\
\hline 6 & & 2.19 & 0.83 & 0.34 & 0.33 & \\
\hline 8 & & 1.38 & 0.76 & 0.51 & 0.30 & \\
\hline 10 & & 1.35 & 1.34 & 0.70 & 0.30 & \\
\hline 12 & & 2.33 & 1.36 & 0.40 & 0.55 & \\
\hline \multirow[t]{2}{*}{14} & & 1.68 & 0.33 & 0.20 & 0.16 & \\
\hline & $\bar{X} \pm S$ & $1.88 \pm 0.47$ & $0.92 \pm 0.39$ & $0.42 \pm 0.17$ & $0.31 \pm 0.14$ & \\
\hline 4 & \multirow{6}{*}{32} & 2.36 & 0.89 & 0.37 & 0.21 & \multirow{6}{*}{0.92} \\
\hline 6 & & 2.32 & 0.66 & 0.53 & 0.25 & \\
\hline 8 & & 2.32 & 0.73 & 0.48 & 0.42 & \\
\hline 10 & & 2.20 & 0.50 & 0.41 & 0.34 & \\
\hline 12 & & 1.99 & 0.78 & 0.51 & 0.45 & \\
\hline \multirow[t]{3}{*}{14} & & 2.68 & 0.33 & 0.22 & 0.30 & \\
\hline & $\bar{X} \pm S$ & $2.31 \pm 0.23$ & $0.65 \pm 0.20$ & $0.42 \pm 0.12$ & $0.33 \pm 0.09$ & \\
\hline & Total & $1.81 \pm 0.47$ & $0.87 \pm 0.30$ & $0.44 \pm 0.13$ & $0.30 \pm 0.13$ & \\
\hline
\end{tabular}

62 
acelerada en animales de menor peso (Suárez Álvarez y Oquendo Pérez 2012). El mayor consumo de OD se observó en los primeros 10 minutos en todos los pesos de los camarones, aumentando este consumo al incrementar la salinidad. A medida que se incrementó el tiempo de exposición de 10 a 20 min sin contar con una fuente externa de oxígeno, el consumo se redujo de 1.81 a $0.87 \mathrm{mg} / \mathrm{L}$, y de los 30 a 40 minutos, los valores de consumo encontrados en promedio fueron de 0.44 y $0.30 \mathrm{mg} / \mathrm{L}$ de oxígeno, respectivamente. El consumo de OD es una respuesta fisiológica relacionada con el ambiente. La tasa de respiración está vinculada con el metabolismo y el flujo de energía que los camarones regulan hacia los mecanismos de control homeostático (Salvato et al. 2001).

En la acuícultura, se puede cuantificar la tasa de respiración de la columna de agua de un estanque, siendo los mayores consumidores del OD la respiración del fondo y el fitoplancton, $\mathrm{y}$, en menor proporción, el cultivo. Durante un ciclo de producción, las exposiciones a las bajas concentraciones de oxígeno disuelto normalmente son provocadas por tener unidades productivas con poca profundidad, la sobrealimentación, el exceso de materia orgánica y una alta productividad primaria. La hipoxia, que es la baja concentración de OD, afecta la conducta y fisiología del camarón (Ulaje Fernández 2014). Cuando la disponibilidad del oxígeno en el cuerpo de agua es menor de $2 \mathrm{mg} / \mathrm{L}$, los organismos presentan un estrés fisiológico, inapetencia, susceptibilidad a enfermedades, rechazo del alimento, y mortalidades al mantener esta concentración por algunas horas (Ron y Espinoza 2019).

El rango óptimo de OD que un productor debería manejar es entre 4 a $7 \mathrm{mg} / \mathrm{L}$ de oxígeno. Cuando se disminuye estas concentraciones, el camarón gasta más energía al transferir más agua por las branquias (Puente Carreón 2009). Al tener concentraciones de 2 a $3.9 \mathrm{mg} / \mathrm{L}$ de OD, el camarón presenta estrés fisiológico, rechazo del alimento, susceptibilidad a enfermedades, lento crecimiento y baja conversión alimenticia (Ron y Espinoza 2019).

Nonwachai et al. (2011) encontraron que camarones mantenidos a concentraciones inferiores a $2 \mathrm{mg} / \mathrm{L}$ de oxígeno durante 60 días tuvieron un 56 $\%$ de sobrevivencia, menor contenido de hemocianina en la hemolinfa y un $26 \%$ de sobrevivencia al ser sometidos en pruebas de desafío con Vibrio harveyi, en comparación con organismos mantenidos a $4 \mathrm{mg} / \mathrm{L}$ que tuvieron $92 \%$ de sobrevivencia, mayor concentración de hemocianina y un $57 \%$ de sobrevivencia en pruebas de desafío con Vibrio harveyi.

En un estanque de cultivo que no cuenta con el oxígeno que demanda el cuerpo de agua, la tasa de respiración y el metabolismo en los camarones disminuye. En los primeros 20 minutos, consume el 50 $\%$ del oxígeno; a los 40 minutos, se consumen hasta el $92 \%$ del oxígeno disponible. Al aumentar el tiempo sin una fuente de oxígeno, la tasa de respiración y el metabolismo baja hasta llegar a niveles críticos que les provoca la muerte. Por esa razón, es importante manejar adecuadamente la tasa de saturación del oxígeno en el cuerpo de agua.

\section{CONCLUSIONES}

El mayor consumo de oxígeno se observó en los camarones en salinidades de 32 UPS $(\mathrm{P}<0.0001)$, mientras que los organismos que estuvieron a salinidades menores presentaron una menor demanda de oxígeno y la tasa de respiración se redujo, al igual que el proceso osmorregulatorio y consumo energético.

Los organismos expuestos a $31{ }^{\circ} \mathrm{C}$ tuvieron un mayor consumo de $\mathrm{OD}(\mathrm{P}<0.0002)$ con $3.44 \mathrm{mg} / \mathrm{L}$ de oxígeno. A los $27^{\circ} \mathrm{C}$, este fue de $3.17 \mathrm{mg} / \mathrm{L}$. Al igual que la salinidad, la temperatura es un factor ambiental que influye en la tasa de respiración y el metabolismo. Los camarones tienen la habilidad de adaptación a los cambios de temperatura y salinidad. 
El peso de los camarones influyó en el consumo de OD $(\mathrm{P}<0.0001)$. A los 40 minutos, en los organismos de 14 y $12 \mathrm{~g}$, la cantidad de oxígeno consumido fue de 3.76 y $3.91 \mathrm{mg} / \mathrm{L}$, respectivamente; en los camarones de 4 y $6 \mathrm{~g} \mathrm{mg} / \mathrm{L}$, el consumo fue de 3.18 y 3.38 $\mathrm{mg} / \mathrm{L}$, respectivamente. Organismos de mayor peso mostraron una mayor tasa de respiración. La demanda energética para sus procesos metabólicos fue mayor con relación a los camarones de menor tamaño.

En general, a los 10 minutos de exposición sin una fuente externa de oxígeno, los camarones utilizaron la mayor cantidad de OD disponible. Al aumentar el tiempo, la tasa de respiración y el metabolismo se redujo hasta llegar a la muerte. En promedio, se observó que a los 10, 20, 30 y 40 minutos sin oxígeno en el medio, la cantidad consumida de OD fue de 1.81 a $0.87,0.44$ y $0.30 \mathrm{mg} / \mathrm{L}$ de oxígeno, respectivamente. En valores porcentuales, se encontró que a los 20 minutos, los camarones consumieron el $52 \%$ del oxígeno disponible, a los 30 minutos el $75.7 \%$ y a los 40 minutos el $83.2 \%$ del OD disponible.

La cantidad de OD que debe mantenerse en el cultivo del camarón es entre 7 y 4 mg/L. Al reducir la disponibilidad del OD, los camarones gastan hasta el $25 \%$ de su energía para mantener sus procesos homeostáticos, son vulnerables a enfermarse, la tasa de conversión alimenticia es alterada, desarrollan una baja ganancia de peso y presentan estrés fisiológico. Cuando existen muy bajas concentraciones de OD, los organismos rechazan el alimento, son más expuestos al ataque de enfermedades y se presentan mortalidades.

\section{REFERENCIAS BIBLIOGRÁFICAS}

Boyd, C. E. (1990). Water Quality in Ponds for Aquaculture. Alabama Agricultural Experiment Station. Auburn University, Alabama.

Boyd, C. E. (1998). Pond water aeration systems. Aquacultural Engineering, 18(1), 9-40. doi: 10.1016/S0144-8609(98)00019-3
Boyd, C. E. (2014). Water Quality An Introduction ( $2^{\circ}$ ed.). Auburn, Alabama, USA: Springer International Publishing Switzerland 2000. doi: 10.1007/978-3-319-17446-4

Boyd, C. E., \& Tucker, C. S. (1992). Water quality and pond soil analysisforaquaculture.Auburn,Alabama,USA:Department of Fisheries and Allied Aquaculture, Auburn University.

Carranza, E. O. (2018). Manual de producción de fincas: para mejorar la competitividad en el cultivo del camarón. (Funder, Ed.) Tegucigalpa, Francisco Morazán, Honduras.

FIDE (2017). Fundación para la inversión y desarrollo de exportaciones. (F. División de promoción de exportación, Ed.) Recuperado el junio de 2019, de Camarón, ficha N. ${ }^{\circ}$, mercado Unión Europea. Disponible en: http://fidehonduras. com/wp-content/uploads/2018/03/FICHA-No.-8-CamaronFIDE-2017.pdf

Galand, D. P., Ashari, A. K., Sulmatiwi, L., Mahasri, G., \& Sari, L. A. (2019). The oxygen content and dissolved oxygen consumption level of white shrimp Litopenaeus vannamei in the nanobubble cultivation system. IOP Conference Series: Earth and Environmental Science, 236(1), 012014. doi:10.1088/1755-1315/236/1/012014

Nonwachai, T., Purivirojku, W., Chuchird, N., \& Limsuwan, C. (2011). Effects of dissolved oxygens levels on growth, survival and inmune response of juvenile pacific white shrimp. Journal of Fisheries and Environment, 35(3), 1-10

Ponce-Palafox, J. T., Ulloa García, M., Ruíz Luna, A., \& Esparza Leal, H. (2013). A response-surface analysis of the relative importance of the temperature, salinity and body weight on the respiratory metabolism of the white shrimp Litopenaeus vannamei (Boone, 1931). Marine and Freshwater Behaviour and Physiology, 46(6), 399-417. doi:10.1080/10236244.201 3.849058

Puente Carreón, E. (2009). Repuesta fisiológica de juveniles de camarón blanco Litopenaeus vannamei, a condiciones oscilantes de oxígeno disuelto y temperatura (Tesis doctoral). Instituto Politécnico Nacional, Centro Interdisciplinario de Ciencias Marinas, La Paz, Baja California, México

Rodríguez, L. (2019). La exportación de camarón aumenta 6 millones de dólares.(PUBLYNSA, Ed.) El Heraldo. Obtenido

64 
de: https://www.elheraldo.hn/economia/1271296-466/laexportacion-de-camaron-aumenta-6-millones-de-dolares

Ron, E., \& Espinoza, C. (2019). Oxígeno disuelto y porcentaje de saturación: su importancia para el manejo eficiente de la aireación. (Nicovita, Ed.) Lima, Perú. Recuperado noviembre de 2019

Salvato, B., Cuomo, V., Muro, P. D., \& Beltramini, M. (2001). Effects of enviromental parameters on the oxygen consumption of four marine invertebrates: a compartative factorial study. Marine Biology, 138(4), 659-668. doi: $10.1007 / \mathrm{s} 002270000501$

Suárez Álvarez, G., \& Oquendo Pérez, O. (2012). Consumo de oxígeno y punto crítico en langostas Panulirus. Revista Electrónica de Veterinaria, 13(3)

Supriatna, Marsoedi, Hariati, A. M., \& Mahmudi, M. (2017). Dissolved oxygen models in intensive culture of whiteleg shrimp, Litopenaeus vannamei, in East Java, Indonesia. Aquaculture, Aquarium, Conservation \& Legislation International Journal of the Bioflux Society, 10(4), 768-778

Ulaje Fernández, S. A. (2014). Relación entre respuestas fisológicas, contenido bioquímico y expresión del camarón blanco (Litopenaeus vannamei) ante cambios agudos y crónicos de hipertermia e hipoxia (Tesis de maestría). Centro de Investigaciones Biológicas del Noroeste, S.C., La Paz, Baja California, México

Valenzuela-Quiñónez, W., Rodríguez-Quiroz, G., Ponce-Palafox, J. T., \& Esparza-Leal, H. M. (2011). Efecto de diferentes combinaciones de temperatura y salinidad sobre el consumo específico de oxígeno en el camarón blanco Litopenaeus vannamei. Revista de Biología Marina y Oceanografía, 46(3), 303-311. doi: 10.4067/S0718-19572011000300002

Yan, B., Wang, X., \& Cao, M. (2007). Effects of salinity and temperature on survival, growth, and energy budget of juvenile Litopenaeus vannamei. Journal of Shellfish Research, 26(1), 141-146. doi:10.2983/07308000(2007)26[141:EOSATO]2.0.CO;2

YSI (2002). Manual de operación, sistema portable de medición de oxígeno disuelto y temperaruta modelo YSI modelo 55. Yellow Springs, Ohio, Estados Unidos 